\title{
Détermination de limites acceptables pour les incertitudes en radiotoxicologie par une méthode utilisant les résultats d'évaluation externe de la qualité
}

\author{
C. Guichet ${ }^{1,2, *}$ et C. Hurtgen ${ }^{2}$ \\ ${ }^{1}$ CEA/SACLAY DRF/PSE/laboratoire de biologie médicale, 91191 Gif-sur-Yvette, France. \\ 2 Association PROCORAD CEA, bureau du conseiller médical, 18, route du Panorama, BP 16, 92265 Fontenay-aux-Roses, France.
}

Reçu le 1 décembre 2016 / Accepté le 7 mars 2017

\begin{abstract}
Résumé - PROCORAD organise depuis plus de 20 ans des essais d'intercomparaisons en radiotoxicologie afin d'évaluer l'aptitude des laboratoires participants en terme d'exactitude. Les résultats à ces intercomparaisons montrent également de grandes variations dans l'estimation des incertitudes fournies par ces laboratoires. Les obligations réglementaires liées à l'accréditation, qui leur demande de définir des exigences de performances et de suivre régulièrement les estimations des incertitudes, et l'absence de limites acceptables publiées ou réglementaires en radiotoxicologie, ont amené PROCORAD à proposer des limites acceptables d'incertitudes issues de l'état de l'art. PROCORAD a utilisé une nouvelle méthode de calcul des incertitudes fondée uniquement sur les résultats des essais d'intercomparaison : la méthode des incertitudes à long terme (long-term uncertainties method [LTUM]). Cette méthode a été appliquée pour le ${ }^{3} \mathrm{H}$ dans les urines, les émetteurs $\gamma / X$ dans les urines et les actinides dans les cendres de selles. La méthode LTUM peut être utilisée en radiotoxicologie comme une méthode simple et pratique de calcul d'incertitudes. Trois objectifs (optimal, souhaitable et minimal) de limites acceptables d'incertitude ont été déterminées à l'aide des premiers quartiles, médianes et troisièmes quartiles des incertitudes calculées par la méthode LTUM : $10 \%, 15 \%$ et $20 \%$ pour le ${ }^{3} \mathrm{H}$ respectivement, $18 \%, 21 \%$ et $27 \%$ pour les émetteurs $\gamma / X$ respectivement, $15 \%, 25 \%$ et $34 \%$ pour le ${ }^{238} \mathrm{Pu}$ respectivement, $11 \%, 17 \%$ et $26 \%$ pour le ${ }^{239} \mathrm{Pu}$ respectivement, $19 \%, 26 \%$ et $38 \%$ pour $1{ }^{241} \mathrm{Am}$ respectivement et $19 \%, 30 \%$ et $52 \%$ pour le ${ }^{244} \mathrm{Cm}$ respectivement. L'application de la méthode LTUM aux résultats fournis par les laboratoires montre une tendance à une sous-estimation des incertitudes calculées par les laboratoires indiquant que tous les facteurs d'influence ne sont pas toujours pris en compte dans le calcul des incertitudes.
\end{abstract}

\begin{abstract}
Determining of acceptable limits of uncertainties in radiotoxicology by using a method based only on external quality assessment results. PROCORAD organizes for more than 20 years proficiency tests in radiotoxicology to estimate the performance of the laboratories in term of accuracy. The results of these intercomparisons also show a wide range of uncertainties values provided by these laboratories. The regulatory obligations related to accreditation, which require that the laboratory shall define the performance requirements for measurement uncertainty and regularly review estimates of measurement uncertainty, and the lack of published or required acceptable limits in radiotoxicology led PROCORAD to propose uncertainties acceptable limits from the "state of art". For this purpose, PROCORAD used a new method to estimate the uncertainties based only on external quality assessment results: the Long-Term Uncertainties Method (LTUM). This method was applied for tritium in urines, $\gamma / X$ emitters in urines and actinides in fecal ashes. This study allowed validation of LTUM by a simple and reliable method for radiotoxicology uncertainties measurements. Three targets (optimal, desirable and minimal) for acceptable limits were defined by using the first quartiles, medians and third quartiles calculation of uncertainties provided by LTUM: $10 \%, 15 \%$ et $20 \%$ for ${ }^{3} \mathrm{H}$ respectively, $18 \%, 21 \%$ et $27 \%$ for $\gamma / X$ emitters respectively, $15 \%, 25 \%$ et $34 \%$ for ${ }^{238} \mathrm{Pu}$ respectively, $11 \%, 17 \%$ et $26 \%$ for ${ }^{239} \mathrm{Pu}$ respectively, $19 \%, 26 \%$ et $38 \%$ for ${ }^{241} \mathrm{Am}$ respectively and $19 \%, 30 \%$ et $52 \%$ for ${ }^{244} \mathrm{Cm}$ respectively. Applying the LTUM method to the results provided by laboratories shows an underestimation of the uncertainties
\end{abstract}

\footnotetext{
* Auteur de correspondance : claude.guichet@cea.fr
} 
calculated by laboratories, indicating that all influencing factors are not always taken into account in the uncertainties measurements.

Keywords: uncertainties / bioassay / quality assurance

\section{Introduction}

Dans les laboratoires de biologie médicale (LBM), notamment dans les laboratoires de radiotoxicologie, l'évaluation externe de la qualité ou EEQ (évaluation, réalisée par un organisme extérieur, de la qualité des analyses d'un laboratoire sur des échantillons connus de l'organisme extérieur mais inconnus du laboratoire, et de la comparaison de la performance avec celle d'autres laboratoires) a toujours été utilisée depuis de nombreuses années. Les laboratoires de radiotoxicologie ou les laboratoires effectuant des mesures de radionucléides dans l'environnement doivent estimer leurs incertitudes pour les dosages qu'ils réalisent, surtout depuis que différentes organisations internationales comme l'ISO le demandent, notamment par l'intermédiaire de normes : NF EN ISO/CEI 17025:2005 (ISO, 2005) et NF EN ISO 15189:2012 (ISO, 2012). En effet, la norme NF EN ISO/CEI 17025:2005 (ISO, 2005) indique au chapitre 5.4.6.2 que « les laboratoires d'essais doivent aussi posséder et appliquer des procédures pour estimer l'incertitude de mesure ». La norme NF EN ISO 15189:2012 (ISO, 2012) stipule au chapitre 5.5.1.4 que « le laboratoire doit déterminer l'incertitude de mesure de chaque procédure de mesure dans la phase analytique utilisée pour consigner les grandeurs mesurées sur les échantillons des patients. Le laboratoire doit définir les exigences de performances pour l'incertitude de mesure de chaque procédure de mesure, et régulièrement examiner les estimations d'incertitudes de mesures ».

Les sources d'incertitudes affectant le résultat d'une analyse radiotoxicologique ont été décrites par plusieurs auteurs (Tymen et al., 1998 ; Ritt et al., 2001) : incertitude sur le rendement de mesure, elle-même dépendante de l'incertitude liée au bruit de fond, aux fluctuations de comptage, au titre de l'étalon, à la prise d'essai; incertitude sur les courbes d'étalonnage (dépendant de la décroissante radioactive de l'étalon, du modèle de réalisation de la courbe d'étalonnage). Une approche fondée sur l'application de la méthode dite des 5M (Ishikawa, 1980 ; Charki, 2012) a été décrite pour l'estimation des incertitudes pour la mesure des actinides par spectrométrie alpha : pas moins de 14 sources d'incertitudes ont été relevées. La variabilité interopérateurs pour la définition des pics de spectrométrie alpha a été considérée comme négligeable, les erreurs humaines ou les interférences difficilement chiffrables.

Les LBM réalisent des mesures sur plusieurs dizaines de paramètres biologiques ou toxicologiques par des techniques diverses (glycémie, bilan lipidique, numération formule sanguine, marqueurs cancéreux, marqueurs hormonaux, métaux lourds...) automatisées ou pas et ont le choix dans la manière d'aborder l'estimation des incertitudes de mesure. Ils peuvent utiliser celles décrites dans le « Guide to the expression of uncertainty measurement » (ISO, 2008), connu sous l'acronyme GUM, qui établit les règles générales pourévaluer et exprimer les incertitudes. Il est réputé parfois comme étant difficilement applicable car fondé sur la description d'un modèle mathématique dans lequel chaque facteur d'incertitude est évalué séparément, suivie d'une combinaison de chacun de ces facteurs.
Des approches alternatives, comme l'approche dite « empirique », ont été comparées à l'approche modélisée du GUM. Ce travail a été réalisé par un groupe d'expert d'EUROLAB et publié dans un rapport en 2007 (EUROLAB, 2007). Cette nouvelle approche est fondée sur la recherche de la performance complète de la méthode de mesure (connaissance de l'inexactitude ou de l'erreur totale sur une mesure) et les données de cette recherche sont celles obtenues par l'évaluation de la fidélité intermédiaire (validations intralaboratoire, contrôles internes de la qualité) et par l'évaluation de la justesse (validations inter-laboratoire, intercomparaisons ou tests de compétence). Cette méthode combinant résultats des contrôles qualité internes (CIQ) et évaluations externes de la qualité (EEQ) a été proposée dans le rapport d'EUROLAB et est reprise par le document Cofrac SH GTA 14 (Cofrac, 2011). D'autres méthodes de calcul d'incertitude sont proposées et même normalisées, comme par exemple celle décrite dans la norme NF ISO 11929:2010 (ISO, 2010a).

L'incertitude de mesure est également un moyen pour estimer la performance d'une méthode analytique. Des auteurs (Meijer et al., 2002) ont proposé d'exprimer l'incertitude de mesure comme une performance analytique à long terme du laboratoire en utilisant uniquement les résultats des EEQ de trois paramètres d'hémostase obtenus sur une longue période. Le suivi de l'inexactitude ou de l'erreur totale sur plusieurs années permet le suivi de la performance à long terme de la méthode de mesure par le suivi des erreurs aléatoires (sous forme de coefficient de variation de reproductibilité à long terme) et des erreurs systématiques (par le suivi du biais à long terme). Cette méthode fondée sur la mesure de l'incertitude à long terme (LTUM) a été reprise (Matar et al., 2015) au sein de l'organisme d'intercomparaison PROBIOQUAL pour l'appliquer à 23 paramètres de biochimie, 15 paramètres d'immunologie et 5 paramètres d'hémostase. Cette publication a comparé l'approche décrite dans le document Cofrac $\mathrm{SH}$ GTA 14 (Cofrac, 2011) et l'approche de l'incertitude à long terme (LTUM). Matar et al. ont défini la médiane des incertitudes LTUM des laboratoires participants aux EEQ pour chaque paramètre. Les incertitudes calculées par les deux méthodes sont comparables avec une tendance des valeurs des incertitudes calculées selon la méthode LTUM plus faible que les incertitudes calculées selon le document SH GTA 14 (Cofrac, 2011), quels que soient les domaines.

L'examen des résultats présentés par PROCORAD (Association pour la PROmotion du COntrôle qualité en RADiotoxicologie), qui organise des intercomparaisons interlaboratoires en radiotoxicologie depuis plus de 20 ans, montre que les incertitudes fournies par les laboratoires varient de façon conséquente d'un laboratoire à l'autre, suggérant des approches pour le calcul des incertitudes très différentes.

L'utilisation d'une approche de calcul d'incertitude identique pour tous les laboratoires pourra permettre, aussi bien à l'organisateur d'essais d'aptitudes interlaboratoires qu'aux laboratoires participants, de comparer de façon objective les incertitudes de chaque laboratoire. C'est le premier objectif de cet article. 
La norme NF EN ISO 15189:2012 (ISO, 2012) indique bien que "le laboratoire doit définir les exigences de performances de l'incertitude de mesure » c'est-à-dire comparer l'incertitude à des données publiées par des sociétés savantes comme la Société française de biologie clinique (Vassault et al., 1999) ou le Groupe français d'étude sur l'hémostase et la thrombose (GEHT, 2014), des articles scientifiques (Ricos et al., 1999) ou bien des performances d'incertitudes fixées réglementairement, comme cela peut exister par exemple pour la mesure de la plombémie pour des travailleurs exposés à ce toxique (Ministère du Travail, des Relations sociales, de la Famille, de la Solidarité et de la Ville, 2009). Il n'existe aucune donnée équivalente publiée pour la radiotoxicologie. Le deuxième objectif de cet article est de pouvoir proposer des limites acceptables d'incertitudes dans les trois grands domaines de la radiotoxicologie : la mesure $\alpha$, avec la mesure des actinides dans les selles, la mesure $\beta$ avec la mesure du tritium dans les urines et la mesure $\gamma$ avec la mesure des émetteurs $\gamma / X$ dans les urines.

\section{Matériels et méthodes}

\subsection{PROCORAD}

PROCORAD (www.procorad.org) est une association française créée en 1995 par des biologistes exerçant dans le domaine du nucléaire (CEA et AREVA principalement) pour promouvoir les contrôles qualités en radiotoxicologie. PROCORAD, fournisseur d'essais inter-laboratoires au niveau international, (plus de 70 participants et plus de 20 pays représentés en moyenne chaque année), reconnu par le Cofrac (Comité français d'accréditation) et partenaire de l'AIEA (Agence internationale de l'énergie atomique), propose 12 types d'analyses : émetteurs $\alpha$ (actinides, uranium, ${ }^{210} \mathrm{Po}$ ), émetteurs $\beta\left({ }^{3} \mathrm{H},{ }^{14} \mathrm{C},{ }^{35} \mathrm{~S},{ }^{90} \mathrm{Sr}\right)$ et émetteurs $\gamma$ dans des matrices et cendres de selles. Ce programme est proposé une fois par an.

\subsection{Analyse des échantillons et choix des laboratoires}

Les laboratoires reçoivent tous les ans des urines et des cendres de selles : six échantillons pour la mesure du tritium dans les urines, trois échantillons pour la mesure des émetteurs $\gamma / X$ dans les urines et trois échantillons pour la mesure des actinides dans les cendres de selles. Les échantillons pour la mesure $\gamma$ peuvent contenir un ou plusieurs émetteurs $\gamma$ et/ou $X$; de la même manière, les échantillons de cendre de selles peuvent contenir du ${ }^{238} \mathrm{Pu}$ et/ou du ${ }^{239} \mathrm{Pu}$ et/ou de $1^{241} \mathrm{Am}$ et/ou du ${ }^{244} \mathrm{Cm}$. Des tests d'homogénéité et de stabilité sont effectués selon la norme NF ISO 13528 (ISO, 2015) et les résultats obtenus démontrent que les échantillons sont stables et homogènes.

Les échantillons sont expédiés à température ambiante et sont réceptionnés par les laboratoires dans des délais de un à 21 jours en fonction des pays (médiane de durée de transport de trois jours). Les laboratoires traitent leurs échantillons selon leurs procédures internes comme des échantillons de patients. Les principes des méthodes utilisées, les étalons et les réactifs utilisés sont synthétisés dans le Tableau 1. Chaque laboratoire




participant à l'intercomparaison organisée par PROCORAD a un numéro d'anonymat qui change chaque année. Les laboratoires saisissent leurs résultats sur le site Internet de PROCORAD sous leur numéro d'anonymat. Les résultats sont évalués de façon statistique suivant les recommandations de la norme NF ISO 13528 (ISO, 2015).

L'estimation de l'incertitude par la méthode de la performance à long terme a été effectuée avec les résultats des essais d'intercomparaisons des années 2010, 2011, 2012, 2013 et 2014 pour la mesure du tritium dans les urines et pour la mesure des émetteurs $\gamma / X$ dans les urines et avec les résultats des essais d'intercomparaisons des années 2009, 2010, 2011, $2012,2013,2014$ et 2015 pour la mesure des actinides dans les cendres de selles afin d'avoir un nombre suffisant de résultats par actinide.

Les laboratoires participant à cette étude ont été choisis par le nombre décroissant d'intercomparaisons auxquelles ils participent. Les laboratoires sélectionnés participent à au moins deux de ces intercomparaisons. Les laboratoires sont français et étrangers. 34 laboratoires sur 46 participants par an en moyenne ont été sélectionnés pour l'étude sur l'incertitude à long terme des mesures du tritium dans les urines; 29 laboratoires sur 44 participants par an en moyenne pour la mesure des émetteurs $\gamma / X$ dans les urines et 27 laboratoires sur 34 participants par an en moyenne pour la mesure des actinides dans les cendres de selles.

\subsection{Méthode de calcul de l'incertitude à long terme}

La méthode de calcul de l'incertitude à long terme utilise un calcul de régression linéaire (de type $y=b x+a$ ) (Meijer et al., 2002) qui exprime la valeur du laboratoire (y) comme une fonction de la valeur assignée $(x)$ (valeur certifiée ou moyenne robuste des résultats fournis par les laboratoires, correspondant à un niveau de concentration) où « $b$ » est la pente et « $a$ » l'ordonnée à l'origine. La droite d'identité $(y=x)$ entre les valeurs du laboratoire et les valeurs assignées correspond à la situation idéale où chaque valeur fournie par le laboratoire est la valeur assignée (Fig. 1). La droite parallèle à cette droite d'identité passant par l'origine « $a$ » mais ayant une pente $\langle b\rangle$ identique $(b=1)$ est également représentée. Ainsi, pour chaque point, il est possible de visualiser l'erreur totale (ET) ou inexactitude ainsi que ses composantes systématiques et aléatoires. Pour un échantillon de contrôle donné, l'erreur totale est représentée par la distance entre le point correspondant à la valeur trouvée par le laboratoire, et la valeur assignée située sur la droite d'identité $(y=x)$.

Cette erreur totale est composée de l'erreur systématique et de l'erreur aléatoire (EA). L'erreur systématique ou le biais de justesse à ce niveau de concentration (LT Biais) est représentée par la distance verticale entre la droite d'identité et la droite de régression $(y=b x+a)$. Cette erreur systématique est elle-même composée d'une erreur constante (biais constant ou $\mathrm{BC}$ ) et d'une erreur proportionnelle à la concentration (biais proportionnel ou BP). L'erreur constante $\mathrm{BC}$ (figurée par la distance verticale entre les deux droites parallèles, égale au « $a$ » de la droite de régression $y=b x+a$ ) peut être due à un effet matrice ou un effet de technique utilisée. L'erreur proportionnelle BP (figurée par la distance verticale entre la droite parallèle à la droite d'identité $(y=x+a)$ et la droite de régression $y=b x+a)$ peut être due à une erreur d'étalonnage.

L'erreur aléatoire est caractérisée par la distance verticale entre la droite de régression $(y=b x+a)$ et le point représentant la valeur donnée par le laboratoire. La dispersion des points aux différents niveaux de concentration autour de cette droite de régression représente la reproductibilité intra-laboratoire (fidélité intermédiaire). Cette dispersion résulte de différents facteurs de variabilité : étalonnages divers, opérateurs divers, réactifs divers et autres facteurs pendant la période couverte par les différentes mesures et valeurs fournies par le laboratoire lors des différentes campagnes d'EEQ.

L'analyse de la somme des carrés des écarts pour l'ensemble des échantillons de contrôle à différentes concentrations permet d'estimer les différents termes (erreur aléatoire [EA], biais à long terme [LTB], biais constant [BC] et biais proportionnel $[\mathrm{BP}])$. L'erreur systématique à long terme (LTB) et l'erreur aléatoire à long terme (CV à long terme ou LTCV) permettent de calculer l'incertitude de mesure en faisant la somme quadratique de ces deux termes.

$$
E T=\sqrt{\frac{1}{n} \sum_{i=1}^{n}\left(y_{i}-x_{i}\right)^{2}} .
$$

L'erreur totale, qui est aussi l'incertitude, peut être calculée selon l'équation suivante :

$$
E T=\sqrt{(L T C V \%)^{2}+(L T B \%)^{2}} .
$$

Les deux composantes peuvent être dérivées des deux équations (1) et (2) :

$$
\frac{1}{n} \sum_{i=1}^{n}\left(y_{i}-x_{i}\right)^{2}=\frac{1}{n}\left[(b-1)^{2} \times \sum_{i=1}^{n}\left(x_{i}-\bar{x}\right)^{2}+n(\bar{y}-\bar{x})^{2}+\sum_{i=1}^{n}\left(y_{i}-\bar{y}\right)^{2}\right] \text {, }
$$

soit

$\frac{1}{n} \sum_{i=1}^{n}\left(y_{i}-x_{i}\right)^{2}=\left[(b-1)^{2} \bullet \frac{n-1}{n} \bullet S_{x}^{2}+(\bar{y}-\bar{x})^{2}+\frac{n-2}{n} \bullet S_{y / x}^{2}\right]$,

avec :

$-x_{i}$ : les valeurs assignées ;

$-y_{i}:$ les valeurs du laboratoire ;

$-n$ : le nombre de résultats d'EEQ ;

$-b$ : la pente de la droite de régression;

$-\bar{x}$ : la moyenne des valeurs assignées ;

$-\bar{y}$ : la moyenne des valeurs du laboratoire ;

$-S_{x}^{2}$ : la variance des valeurs assignées $x$

$-S_{y} /_{x}:$ l'écart-type résiduel. LTB :

De l'équation (3), on peut calculer le biais à long terme

$$
L T B=\sqrt{\frac{n-1}{n} \bullet(b-1)^{2} \bullet S_{x}^{2}+(\bar{y}-\bar{x})^{2}} .
$$




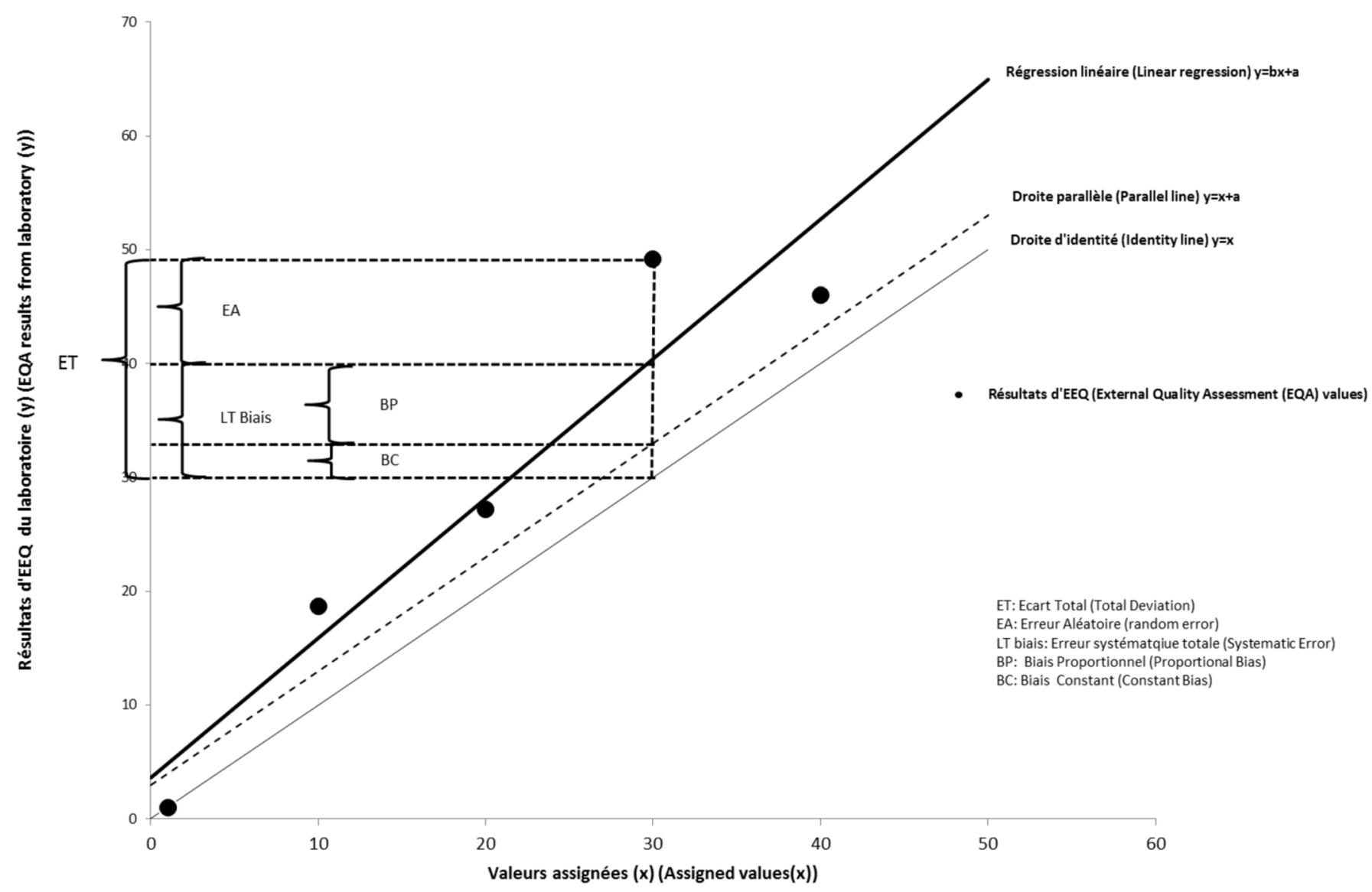

Fig. 1. Modèle de régression linéaire pour l'évaluation de l'incertitude de mesure.

Fig. 1. Linear regression model for assessment of uncertainties measurement.

Pour pouvoir comparer les LTB entre laboratoires, il faut le calculer en pourcentage par rapport à la valeur moyenne des valeurs assignées :

$$
\operatorname{LTB} \%=\frac{\sqrt{\frac{n-1}{n} \bullet(b-1)^{2} \bullet S_{x}^{2}+(\bar{y}-\bar{x})^{2}}}{\bar{x}} \bullet 100 .
$$

Le biais à long terme est composé de la somme quadratique du biais proportionnel (BP) et du biais constant (BC). On peut donc en déduire les équations respectives (6) et (7) :

$$
\begin{gathered}
B P=\sqrt{\frac{n-1}{n} \bullet(b-1)^{2} \bullet S_{x}^{2}}, \\
B C=\sqrt{(\bar{y}-\bar{x})^{2}} .
\end{gathered}
$$

Des équations (1), (2) et (3), on peut déduire l'erreur aléatoire à long terme (écart-type résiduel sans biais) selon l'équation suivante :

$$
E A=\sqrt{\frac{n-2}{n} \cdot S_{y / x}^{2}} .
$$

On peut en déduire le LTCV \% qui est fondé sur l'écarttype résiduel et la moyenne des valeurs assignées. Afin de permettre la comparaison des LTCV entre les laboratoires, il doit être calculé après ajustement par le biais (correction par la pente de la droite de régression linéaire pour être indépendant du biais ou de l'erreur systématique). Le LTCV \% a donc pour équation :

$$
L T C V \%=\frac{\frac{S_{y / x}}{\bar{b}}}{\bar{x}} \bullet 100
$$

Le calcul de l'incertitude élargie à long terme LTUM \%, pour un intervalle de confiance de $95 \%$, peut être déduit des équations (1), (5) et (9) :

\section{$\operatorname{LTUM} \%=$}

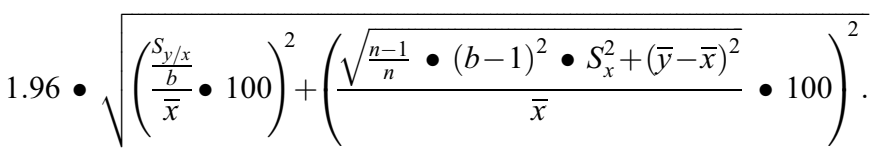

Pour tous les laboratoires choisis pour cette étude, l'incertitude à long terme a été évaluée en utilisant les différentes valeurs assignées obtenues quelles que soient les méthodes (résultats de tous les participants). Le calcul du nombre d'échantillons testés, du LTCV \%, du LTB \% et de l'incertitude à long terme LTUM \% a été réalisé à l'aide du 


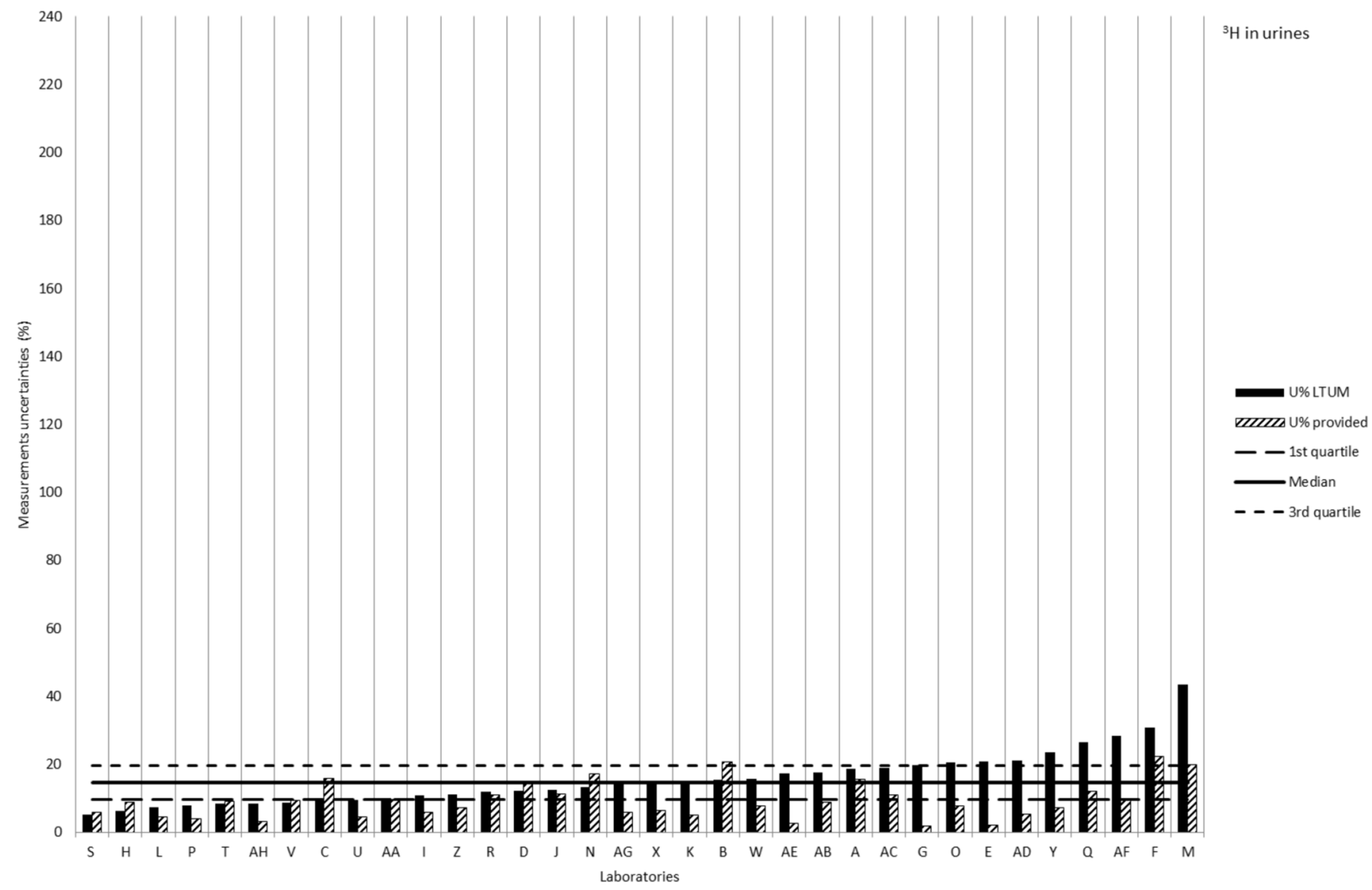

Fig. 2. Comparaison entre les incertitudes calculées selon la méthode LTUM ( $U$ \% LTUM) et des incertitudes moyennes fournies par les laboratoires participants ( $U \%$ provided $)\left({ }^{3} \mathrm{H}\right.$ urinaire) .

Fig. 2. Comparison LTUM uncertainties measurements (U\% LTUM) versus uncertainties measurement provided by each laboratory (U\% provided) (tritium in urines).

Tableau 2. Médianes des incertitudes calculées selon la méthode LTUM et des incertitudes moyennes fournies par les laboratoires distribution Q1 suivant les différents laboratoires).

Table 2. Medians of calculated LTUM uncertainties and medians of average uncertainties provided by laboratories (distribution according to the different laboratories).

\begin{tabular}{|c|c|c|c|c|c|c|c|c|c|c|c|c|}
\hline & \multicolumn{12}{|c|}{ Incertitude élargie $U(\%)$} \\
\hline & \multicolumn{2}{|c|}{${ }^{3} \mathrm{H}(n=34)$} & \multicolumn{2}{|c|}{$\gamma / X(n=29)$} & \multicolumn{2}{|c|}{${ }^{238} \mathrm{Pu}(n=27)$} & \multicolumn{2}{|c|}{${ }^{239} \mathrm{Pu}(n=28)$} & \multicolumn{2}{|c|}{${ }^{241} \mathrm{Am}(n=26)$} & \multicolumn{2}{|c|}{${ }^{244} \mathrm{Cm}(n=27)$} \\
\hline & LTUM & Participants & LTUM & Participants & LTUM & Participants & LTUM & Participants & LTUM & Participants & LTUM & Participants \\
\hline $1^{\mathrm{er}}$ quartile & 10 & 5,7 & 18 & 8,2 & 15 & 11,9 & 11 & 10,1 & 19 & 10,2 & 19 & 11,8 \\
\hline Médiane & 15 & 8,3 & 21 & 11,4 & 25 & 14,0 & 17 & 11,2 & 26 & 14,3 & 30 & 14,5 \\
\hline $3^{\mathrm{e}}$ quartile & 20 & 11,2 & 27 & 15,1 & 34 & 17,0 & 26 & 14,1 & 38 & 15,7 & 52 & 16,7 \\
\hline Minimum & 5,3 & 1,9 & 12,5 & 5,5 & 6,0 & 6,5 & 5,1 & 5,7 & 9,9 & 8,9 & 9,5 & 9,8 \\
\hline Maximum & 43,5 & 22,3 & 60,4 & 25,4 & 76,9 & 29,2 & 81,3 & 22,0 & 50,6 & 24,3 & 220,6 & 22,0 \\
\hline
\end{tabular}

logiciel Excel ${ }^{\circledR}$ v2010. Pour chaque campagne et chaque participant, les valeurs aberrantes ont été exclues (z-score $>3$ à partir des campagnes de 2013 et test de Grubbs pour les années antérieures à 2013). L'estimation de l'incertitude à long terme a été réalisée à partir de six résultats par intercomparaison pour un laboratoire donné.

\subsection{Incertitudes fournies par les laboratoires}

L'étude des incertitudes fournies par les laboratoires sélectionnés montre des différences entre laboratoires et, pour un même laboratoire, des différences ou non au cours du temps, laissant supposer une utilisation de différentes 


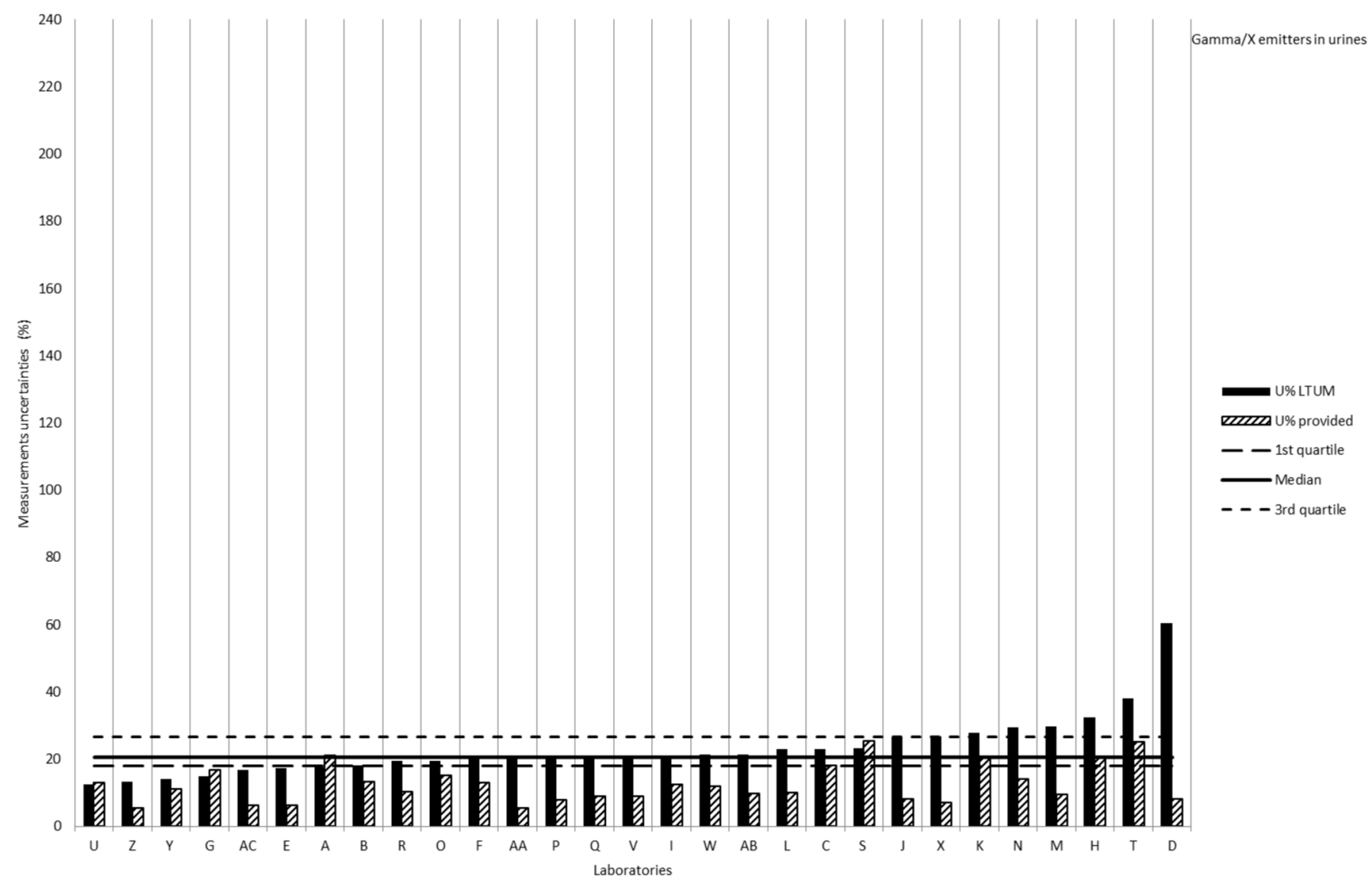

Fig. 3. Comparaison entre les incertitudes calculées selon la méthode LTUM ( $U$ \% LTUM) et des incertitudes moyennes fournies par les laboratoires participants ( $U \%$ provided $)(\gamma / X$ urinaires).

Fig. 3. Comparison LTUM uncertainties measurements (U\% LTUM) versus uncertainties measurement provided by each laboratory (U\% provided) ( $\gamma / \mathrm{X}$ emitters in urine).

approches pour le calcul d'incertitudes. Pour cette étude, nous avons comparé l'incertitude à long terme calculée pour un laboratoire à la moyenne des incertitudes obtenues pour ce laboratoire. PROCORAD n'a jamais demandé la méthode de calcul des incertitudes aux participants.

\section{Résultats}

\subsection{Valeurs des incertitudes selon la méthode LTUM et les incertitudes fournies par les laboratoires participants}

Les résultats des laboratoires participants aux intercomparaisons ont été utilisés pour le calcul de l'incertitude à long terme de chaque laboratoire selon l'équation (8). Une seule valeur assignée a été utilisée, celle de tous les participants car il n'y a pas eu d'analyse par groupe de pairs, PROCORAD ne fournissant pas encore de résultats par groupe de pairs. La distribution des valeurs d'incertitudes à long terme LTUM, obtenues pour tous les laboratoires participants sélectionnés pour cette étude, a été évaluée par le premier quartile, la médiane et le troisième quartile, ainsi que par la valeur minimale et la valeur maximale observée (Tab. 2). La même approche a été appliquée pour les incertitudes moyennes fournies par les laboratoires, ce qui permet de comparer l'estimation des incertitudes par les deux méthodes.

La médiane des incertitudes à long terme varie, suivant le type d'analyse, de $14,8 \%$ pour le ${ }^{3} \mathrm{H}$ dans les urines jusqu'à $30 \%$ pour le ${ }^{244} \mathrm{Cm}$ dans les cendres de selles. La médiane des incertitudes fournies par les laboratoires varie de $8,3 \%$ pour le ${ }^{3} \mathrm{H}$ à $14 \%$ pour trois actinides dans les cendres de selles, soit une sous-estimation d'un facteur $1,5\left({ }^{239} \mathrm{Pu}\right)$ à $2,1\left({ }^{244} \mathrm{Cm}\right)$ (Tab. 2). Pour chaque série d'intercomparaison, en analysant deux à deux, pour chaque laboratoire participant, les incertitudes LTUM et les incertitudes fournies par les laboratoires participants, il apparaît qu'une majorité de laboratoires sous-estime ses incertitudes, quel que soit le niveau d'incertitude fournie ou calculée (Figs. 2-7).

Afin de ne pas dépendre du niveau de la valeur de l'incertitude, les rapports des incertitudes entre elles ont été calculés et comparés à deux types de limites acceptables, permettant d'estimer le niveau d'évaluation de l'incertitude calculée par le laboratoire par rapport à l'incertitude LTUM. Le premier niveau de limites acceptables, restrictif, fixe comme limite supérieure un rapport égal à 1,5 (incertitude LTUM supérieure de $50 \%$ à l'incertitude calculée). Plus de $59 \%$ des laboratoires sous estiment leurs incertitudes pour tous les types d'examen hormis le ${ }^{239} \mathrm{Pu}$. Dans le cas du ${ }^{239} \mathrm{Pu}, 43 \%$ des 


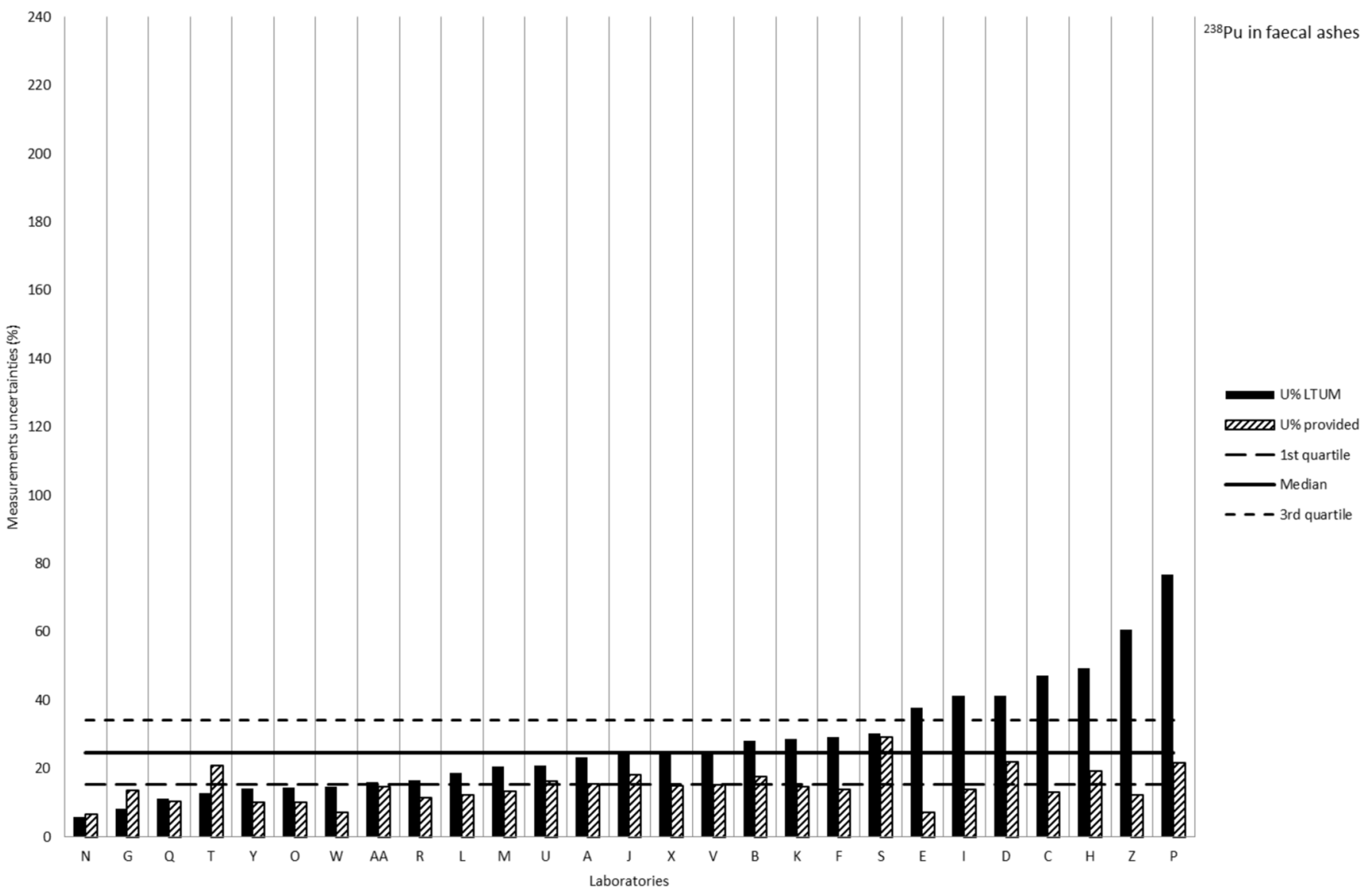

Fig. 4. Comparaison entre les incertitudes calculées selon la méthode LTUM ( $U$ \% LTUM) et des incertitudes moyennes fournies par les laboratoires participants ( $U \%$ provided) $\left({ }^{238} \mathrm{Pu}\right.$ dans les cendres de selles).

Fig. 4. Comparison LTUM uncertainties measurements (U\% LTUM) versus uncertainties measurement provided by each laboratory (U\% provided) $\left({ }^{238} \mathrm{Pu}\right.$ in fecal ashes).

Tableau 3. Niveau d'estimation des incertitudes.

Table 3. Level of uncertainties estimation.

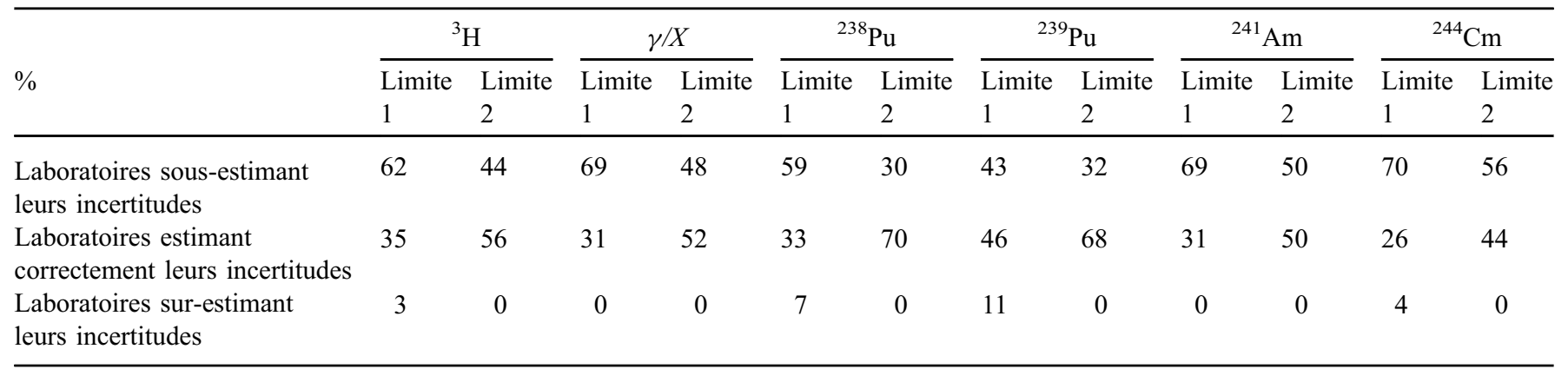

Limite 1 : rapport incertitudes calculées/incertitudes LT ou incertitude LT/incertitudes calculées > 1,5; limite 2 : rapport incertitudes calculées/ incertitudes LT ou incertitude LT/incertitudes calculées $>2$.

laboratoires sous estiment leurs incertitudes (Tab. 3). Environ un tiers des laboratoires estiment correctement leurs incertitudes pour tous les paramètres hormis 1 le ${ }^{239} \mathrm{Pu}$; près de la moitié des laboratoires estiment correctement leurs incertitudes pour le ${ }^{239} \mathrm{Pu}$. Le second niveau des limites acceptables, moins restrictif, fixe comme limite supérieure un rapport égal à 2 (incertitude calculée supérieure ou inférieure de $100 \%$ à l'incertitude LTUM). En prenant cette limite, environ la moitié des laboratoires estiment correctement leurs incertitudes pour tous les paramètres hormis les plutoniums dans les cendres de selles pour lesquels environ deux tiers des laboratoires estiment correctement leurs incertitudes (Tab. 3). Les rapports 


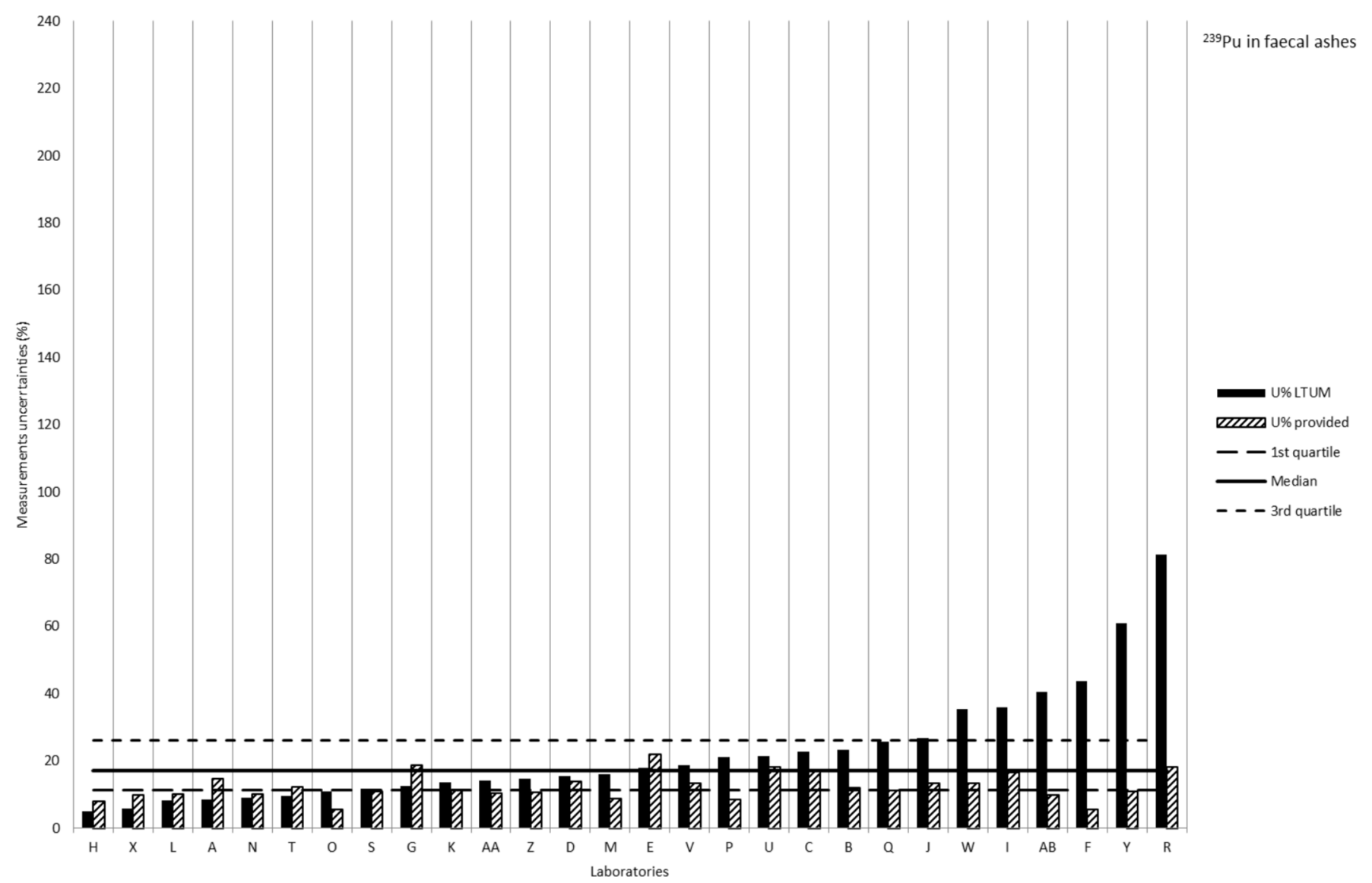

Fig. 5. Comparaison entre les incertitudes calculées selon la méthode LTUM ( $U$ \% LTUM) et des incertitudes moyennes fournies par les laboratoires participants ( $U \%$ provided) $\left({ }^{239} \mathrm{Pu}\right.$ dans les cendres de selles).

Fig. 5. Comparison LTUM uncertainties measurements (U\% LTUM) versus uncertainties measurement provided by each laboratory (U\% provided) ( ${ }^{239} \mathrm{Pu}$ in fecal ashes).

entre les incertitudes fournies par les laboratoires et les incertitudes calculées selon la méthode LTUM varient de 0,6 à 10,4 pour le ${ }^{3} \mathrm{H}$ dans les urines, de 0,8 à 7,4 pour les émetteurs $\gamma / X$ dans les urines et de 0,6 à 5,2 pour $l{ }^{238} \mathrm{Pu}$, de 0,6 à 7,7 pour le ${ }^{239} \mathrm{Pu}$, de 0,7 à 4,7 pour $1{ }^{2241}$ Am et de 0,8 à 12,4 pour le ${ }^{244} \mathrm{Cm}$ (Figs. 8-10).

\subsection{Paramètres prépondérants du calcul d'incertitudes selon la méthode LTUM}

L'incertitude LTUM est composée selon les équations (1) et (8) par le biais à long terme (LTB) et le coefficient de variation à long terme (LTCV). En analysant chacun de ces deux termes, on peut évaluer lequel est le plus important dans l'estimation de cette incertitude. Nous avons considéré que, lorsque le rapport entre les deux termes était supérieur à 2, l'un des termes devenait prépondérant. Pour la mesure $\mathrm{du}^{3} \mathrm{H}$ dans les urines, les émetteurs $\gamma / X$ dans les urines, le ${ }^{239} \mathrm{Pu}$ et le ${ }^{244} \mathrm{Cm}$ dans les cendres de selles, il apparaît que c'est le facteur lié à la fidélité intermédiaire, c'est-à-dire le terme LTCV qui est prépondérant dans le budget du calcul d'incertitudes pour $32,4 \%, 27,6 \%, 39,3 \%$ et $25,9 \%$ des laboratoires respectivement. Pour les autres laboratoires, il n'y a pas de prépondérance d'un terme par rapport à l'autre. Par contre, pour le ${ }^{238} \mathrm{Pu}$, c'est le facteur lié à la justesse, c'est-à-dire le terme LTB qui est prépondérant pour $44 \%$ des laboratoires. Pour $1^{, 241} \mathrm{Am}$, il y a autant de laboratoires pour lesquels le facteur prépondérant dans le calcul de l'incertitude est lié à la fidélité intermédiaire que de laboratoires pour lesquels le facteur prépondérant est lié à la justesse (Tab. 4).

\section{Discussion}

Le premier but de ce travail était de valider une méthode simple et pratique de calcul d'incertitude pour les laboratoires de radiotoxicologie en utilisant uniquement les résultats des évaluations externes de la qualité sans avoir besoin des résultats de contrôle qualité interne ou de tout autre paramètre nécessaire pour l'estimation des incertitudes. Le second objectif est de proposer des limites acceptables d'incertitude auxquelles pourront se comparer les laboratoires de radiotoxicologie afin d'améliorer leurs performances.

Nous avons estimé pour cela l'incertitude à long terme de six types d'analyse de la radiotoxicologie en utilisant les résultats sur cinq ans du circuit d'intercomparaison PROCORAD : ${ }^{3} \mathrm{H}$ dans les urines, les $\gamma / X$ dans les urines, les actinides $\left({ }^{238} \mathrm{Pu},{ }^{239} \mathrm{Pu},{ }^{241} \mathrm{Am}\right.$ et $\left.{ }^{244} \mathrm{Cm}\right)$ dans les cendres de selles. 


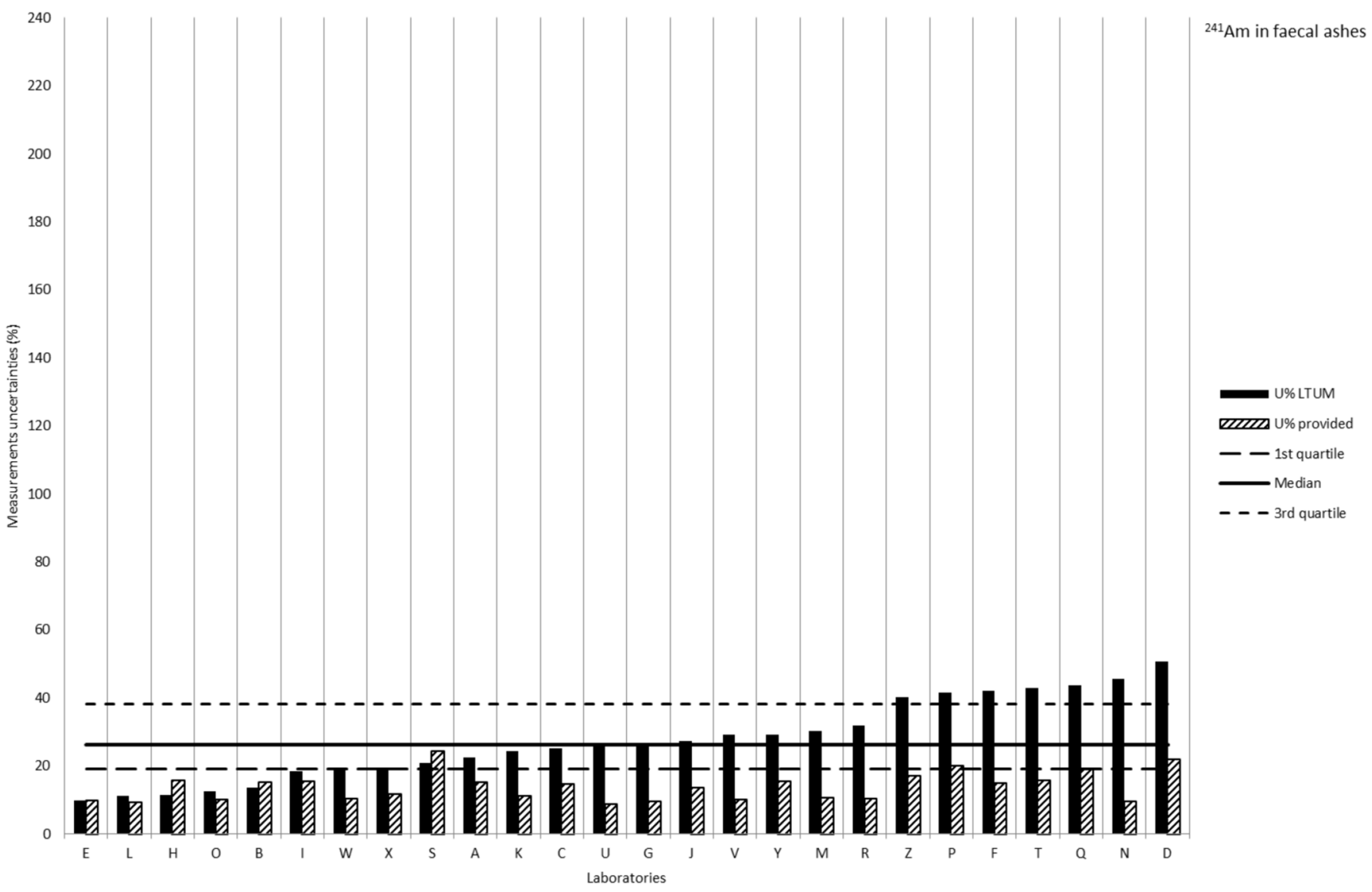

Fig. 6. Comparaison entre les incertitudes calculées selon la méthode LTUM ( $U$ \% LTUM) et des incertitudes moyennes fournies par les laboratoires participants ( $U \%$ provided) $\left({ }^{241} \mathrm{Am}\right.$ dans les cendres de selles).

Fig. 6. Comparison LTUM uncertainties measurements (U\% LTUM) versus uncertainties measurement provided by each laboratory (U\% provided) $\left({ }^{241} \mathrm{Am}\right.$ in fecal ashes).

Tableau 4. Composantes de l'incertitude : proportion de laboratoires dont l'une des composantes est prépondérante (rapport LTCV/LTB ou $\mathrm{LTB} / \mathrm{LTCV}>2$ ).

Table 4. Uncertainties components: proportion of laboratories including one of the components is predominant (ratio LTCV/LTB or LTB/ $L T C V>2)$.

\begin{tabular}{lrrrrr}
\hline$\%$ & ${ }^{3} \mathrm{H}$ & $\gamma / X$ & ${ }^{238} \mathrm{Pu}$ & ${ }^{239} \mathrm{Pu}$ & ${ }^{241} \mathrm{Am}$ \\
\hline Incertitude liée à la fidélité intermédiaire (CV à long terme LTCV) & 32,4 & 27,6 & 7,4 & 39,3 & 26,9 \\
Incertitude liée à la justesse (biais à long terme LTB) & 0,0 & 3,4 & 44,4 & 7,1 & 30,8 \\
\hline
\end{tabular}

L'approche de l'incertitude par la méthode de l'incertitude à long terme est fondée sur l'étude de la régression linéaire entre les résultats du laboratoire et les valeurs assignées fournies par les organisateurs d'essais d'intercomparaisons. En outre, les paramètres de la droite de régression (pente et ordonnée à l'origine) sont des estimations du biais proportionnel et $\mathrm{du}$ biais constant permettant l'utilisation d'échantillons de contrôle à différents niveaux d'activité. La qualité de ces estimations est d'autant meilleure que le nombre de degré de liberté augmente, dépendant du nombre d'échantillons de contrôle pris en compte.
Un certain nombre de laboratoires propose une incertitude élargie proportionnelle au niveau d'activité, accordant une part prépondérante, dans le calcul de l'incertitude élargie, à l'incertitude liée au taux de comptage suivant la loi de Poisson. D'autres laboratoires proposent une incertitude constante quel que soit le niveau d'activité, indiquant des approches très variées dans le calcul des incertitudes. PROCORAD n'a pas d'informations de la part des laboratoires pour savoir s'ils utilisent la méthode GUM ou une méthode proche de celle du SH GTA 14 (utilisation des résultats des CIQ et EEQ) ou bien une toute autre méthode. Une évaluation 


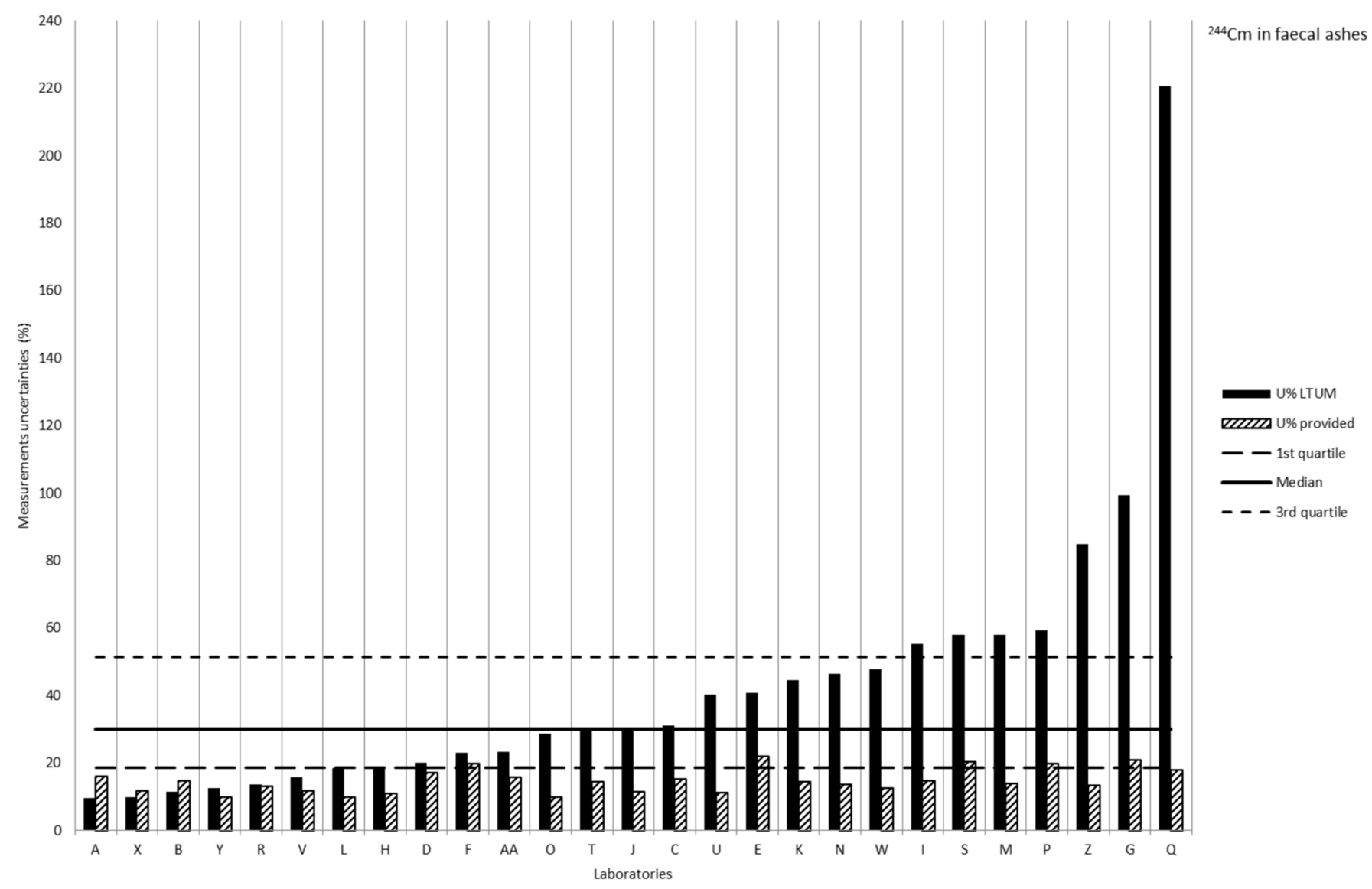

Fig. 7. Comparaison entre les incertitudes calculées selon la méthode LTUM ( $U$ \% LTUM) et des incertitudes moyennes fournies par les laboratoires participants ( $U \%$ provided $)\left({ }^{244} \mathrm{Cm}\right.$ dans les cendres de selles).

Fig. 7. Comparison LTUM uncertainties measurements (U\% LTUM) versus uncertainties measurement provided by each laboratory (U\% provided) $\left({ }^{244} \mathrm{Cm}\right.$ in fecal ashes).

de la méthode utilisée peut être cependant approchée par la connaissance des profils des laboratoires participants. Il y a une majorité de laboratoires travaillant dans l'environnement et la recherche, pour la plupart accrédités selon la norme NF EN ISO/CEI 17025 (ISO, 2005). Ces laboratoires devraient avoir une approche GUM dans la majorité des cas, pouvant expliquer les différences rencontrées avec la méthode LTUM. De ce fait, la comparaison des incertitudes 2 à 2 pour un même laboratoire peut être difficile à interpréter.

La comparaison des médianes d'incertitudes de calcul selon la méthode LTUM avec les médianes des incertitudes moyennes fournies par les laboratoires montre que les laboratoires participants sous-estiment leurs incertitudes quel que soit le type d'analyse. Environ 2/3 des laboratoires sousestiment ses incertitudes si on prend le critère de rapport restrictif de 1,5. Il n'y a plus que la moitié des laboratoires qui sous-estiment ses incertitudes avec le critère de rapport moins restrictif de 2. Et il n'y a plus qu'un tiers des laboratoires mesurant les isotopes du plutonium qui a tendance à sousestimer ses incertitudes. On retrouve en revanche une surestimation de l'incertitude fournie par les laboratoires pour les niveaux bas d'activité (résultats non montrés), surtout pour les mesures de ${ }^{3} \mathrm{H}$ dans les urines et des actinides dans les cendres de selles. Cela peut suggérer une prépondérance de la composante liée au biais proportionnel dans l'évaluation de l'incertitude. Cette observation est moins vérifiée pour les émetteurs $\gamma / X$ dans les urines. Certains laboratoires prennent en compte le type d'émission ou l'énergie de la raie émettrice pour pondérer leur incertitude : différence entre émetteur $\gamma$ et émetteur $X$ quel que soit le niveau d'activité présente dans l'échantillon (résultats non montrés).

Des hypothèses doivent être respectées pour valider l'estimation de l'incertitude à long terme : linéarité entre les valeurs de mesures des échantillons d'EEQ rendues par les laboratoires participants et les valeurs assignées fournies par les organisateurs, égalité des variances dans la gamme d'activité étudiée et commutabilité des échantillons de contrôle.

La méthode LTUM a l'avantage de prendre en compte totalement l'évolution du biais en fonction de la concentration. Elle fournit une estimation unique de la variabilité de la régression linéaire pour toute la gamme de concentration explorée par les échantillons de contrôle, tandis que l'écarttype varie avec la concentration ou l'activité. Par conséquent, l'estimation de la variance obtenue par cette méthode pourrait être fiable pour des radionucléides dont la gamme de 


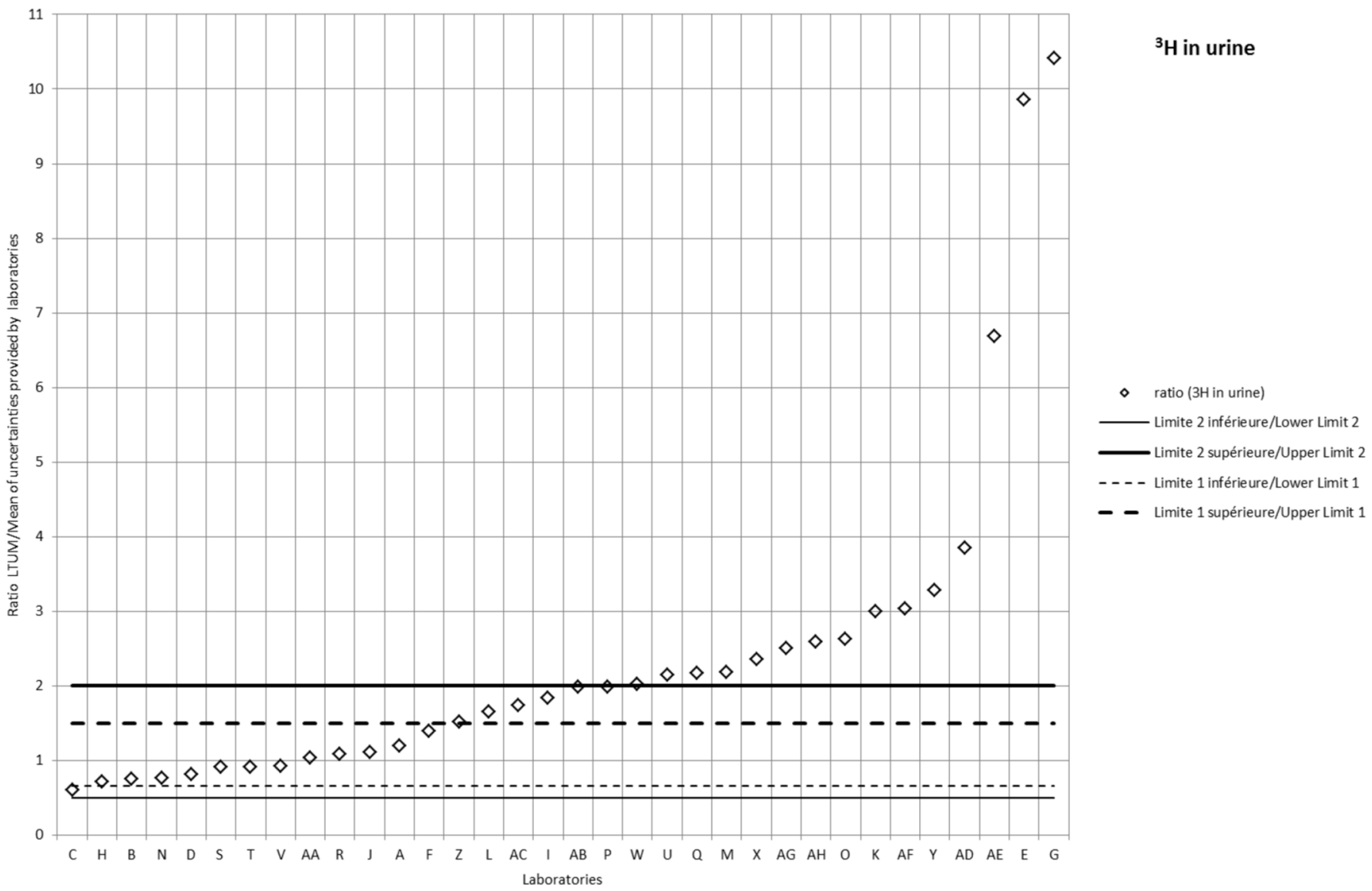

Fig. 8. Rapports entre l'incertitude LTUM et l'incertitude moyenne fournie par les laboratoires $\left({ }^{3} \mathrm{H}\right.$ urinaire).

Fig. 8. Ratio between LTUM uncertainties and average uncertainties provided by laboratories (tritium in urines).

concentration ou d'activité n'excède pas trop les niveaux testés par les essais d'intercomparaisons. Dans le cas des niveaux très élevés de concentration ou d'activité, la méthode LTUM devrait être appliquée en regroupant les échantillons de contrôle en fonction de leur niveau de concentration. Les échantillons de haut niveau de concentration ne sont pas proposés par PROCORAD pour des raisons de réglementations des transports et pour être en adéquation avec les valeurs pouvant être retrouvées dans les échantillons biologiques ou environnementaux analysés par les différents laboratoires. Le calcul d'incertitude par la méthode LTUM devrait être réévalué dans chaque laboratoire notamment en cas d'incident à niveau de contamination élevée.

La commutabilité des échantillons de contrôle par rapport aux échantillons de patient est un paramètre important pour l'estimation de l'incertitude de mesure. C'est à cette condition que le biais, estimé par la méthode LTUM, sera fiable et reflétant uniquement les divergences entre les processus de mesures et non les différences liées à la matrice. Les échantillons expédiés par PROCORAD sont très proches de ceux rencontrés pour les patients suivis dans le cas des risques professionnels. En effet, les urines sont traitées pour être conservées puis surchargées. Les selles sont surchargées après calcination. Le traitement subi par les urines pour le dosage $\mathrm{du}{ }^{3} \mathrm{H}$ urinaire induit un assombrissement significatif de l'échantillon qui n'est pas rencontré en routine dans les laboratoires de biologie médicale ou de l'environnement. Cette différence peut influencer le biais à long terme dans le cas des procédures de mesure en scintillation liquide ne tenant pas compte du quenching couleur. Cependant, parmi les échantillons de ${ }^{3} \mathrm{H}$ urinaires testés, il y a chaque année une urine de patient surveillé qui subit le même traitement physicochimique et qui n'est pas surchargée. Afin d'évaluer la commutabilité des échantillons de contrôle, il faudrait séparer les résultats obtenus par chaque laboratoire pour les échantillons de patients surveillés des échantillons surchargés et recalculer l'incertitude LTUM des deux groupes ainsi différenciés. Il est nécessaire d'avoir au moins six résultats pour faire le calcul de l'incertitude LTUM, ce qui nécessite d'inclure au moins une ou deux années de résultats d'essais d'intercomparaison supplémentaires. Néanmoins, les résultats fournis par les laboratoires sur plusieurs années ne montrent pas de différences significatives entre les échantillons surchargés et les urines de patients. Et afin de prouver la commutabilité des échantillons de contrôle dans chaque type d'EEQ, il faudrait utiliser les procédures décrites par le Clinical and Laboratory Standards Institute (CLSI, 2010). Nous n'avons pas testé la commutabilité des échantillons de contrôle. En outre, l'objectif principal de ce travail est de tester une méthode pratique et simple de calcul d'incertitude et de la 


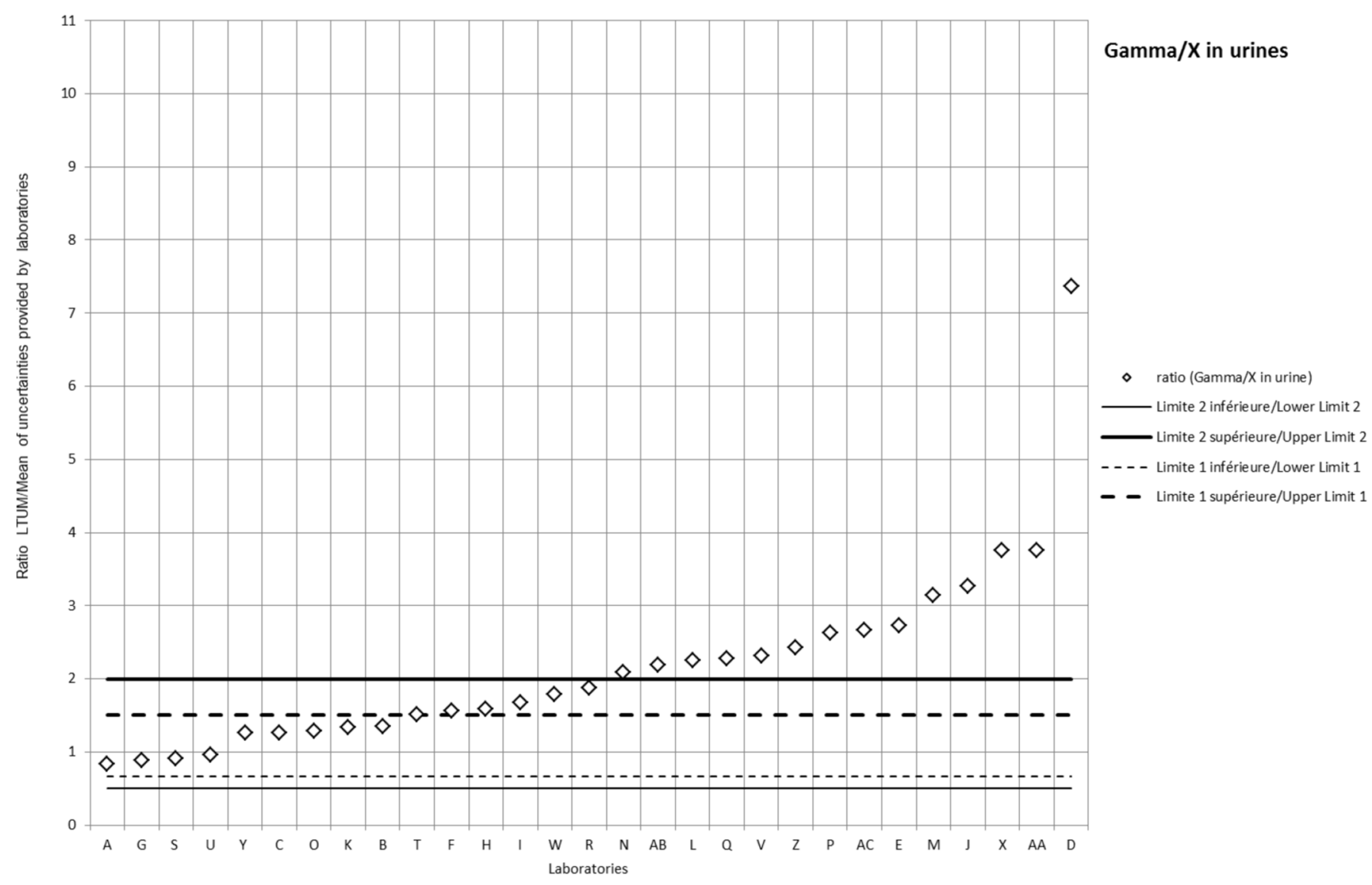

Fig. 9. Rapports entre l'incertitude LTUM et l'incertitude moyenne fournie par les laboratoires ( $\gamma / X$ urinaires).

Fig. 9. Ratio between LTUM uncertainties and average uncertainties provided by laboratories ( $\gamma / \mathrm{X}$ emitters in urine).

comparer à des méthodes recommandées ou existantes qui nécessitent aussi une commutabilité des échantillons.

La méthode LTUM peut également participer à la démarche d'amélioration des laboratoires puisque l'analyse des deux termes contribuant au calcul peut conduire à mettre en place des actions correctives. En effet, si l'analyse montre que l'incertitude de mesure est surtout liée au terme du CV long terme, il peut être intéressant de chercher les causes, en routine, de cette variabilité : variabilité des étalons, variabilité des réactifs, manque d'homogénéité de pratique entre opérateurs, incidence des opérations de maintenance, etc. De la même façon, lorsque l'analyse des paramètres influençant l'incertitude LTUM montre que c'est le terme lié au biais LT qui est prépondérant, il est intéressant pour le laboratoire d'estimer un éventuel effet matrice, de réévaluer la méthode utilisée par rapport aux autres participants, de revoir l'étalonnage ou la valeur de l'étalon interne, etc.

Le deuxième objectif de ce travail est de proposer des limites acceptables d'incertitude de mesure dans le domaine de la radiotoxicologie. La norme NF EN ISO/CEI 17025 (ISO, 2005) indique au chapitre 5.4.6.2 «qu'une estimation raisonnable doit se baser sur une connaissance de la performance de la méthode et sur le domaine de la mesure et faire appel, par exemple, à l'expérience acquise et aux données de validation antérieures ». En revanche, la norme ISO 28218 (ISO, 2010b) portant sur les critères de performances pour les analyses de radiotoxicologie ne fournit que des critères pour le biais $(-25 \%$ à $+50 \%$ par rapport à la valeur assignée) et la répétabilité de ce biais pour cinq mesures minimum (écart-type du biais $<0,4$ ) et pas de critères pour les incertitudes.

Nous avons choisi de proposer comme limites acceptables trois niveaux : un niveau minimal de limites acceptables correspondant au troisième quartile des incertitudes calculées par la méthode LTUM soit $20 \%$ pour $1 \mathrm{l}^{3} \mathrm{H}$ urinaire, $27 \%$ pour les émetteurs $\gamma / X$ dans les urines et pour les actinides dans les cendres de selles, $34 \%$ pour le ${ }^{238} \mathrm{Pu}, 26 \%$ pour le ${ }^{239} \mathrm{Pu}, 38 \%$ pour $\mathrm{l}^{241} \mathrm{Am}$ et $52 \%$ pour le ${ }^{244} \mathrm{Cm}$; un niveau souhaitable correspondant aux médianes des incertitudes calculées par la méthode LTUM soit $15 \%$ pour $1 \mathrm{le}^{3} \mathrm{H}$ urinaire, $21 \%$ pour les émetteurs $\gamma / X$ dans les urines et pour les actinides dans les cendres de selles, $25 \%$ pour le ${ }^{238} \mathrm{Pu}, 17 \%$ pour le ${ }^{239} \mathrm{Pu}, 26 \%$ pour $1^{, 241} \mathrm{Am}$ et $30 \%$ pour le ${ }^{244} \mathrm{Cm}$; un niveau optimal correspondant au premier quartile des incertitudes calculées par la méthode LTUM soit $10 \%$ pour le ${ }^{3} \mathrm{H}$ urinaire, $18 \%$ pour les émetteurs $\gamma / X$ dans les urines et pour les actinides dans les cendres de selles, $15 \%$ pour le ${ }^{238} \mathrm{Pu}, 11 \%$ pour $1 \mathrm{l}^{239} \mathrm{Pu}, 19 \%$ pour $l^{241} \mathrm{Am}$ et $19 \%$ pour le ${ }^{244} \mathrm{Cm}$.

Il est nécessaire de faire une distinction entre les différents actinides du fait des techniques analytiques employées : étapes de radiochimie plus ou moins complexe pour séparer le groupe plutonium du groupe Américium/Curium des autres actinides, 


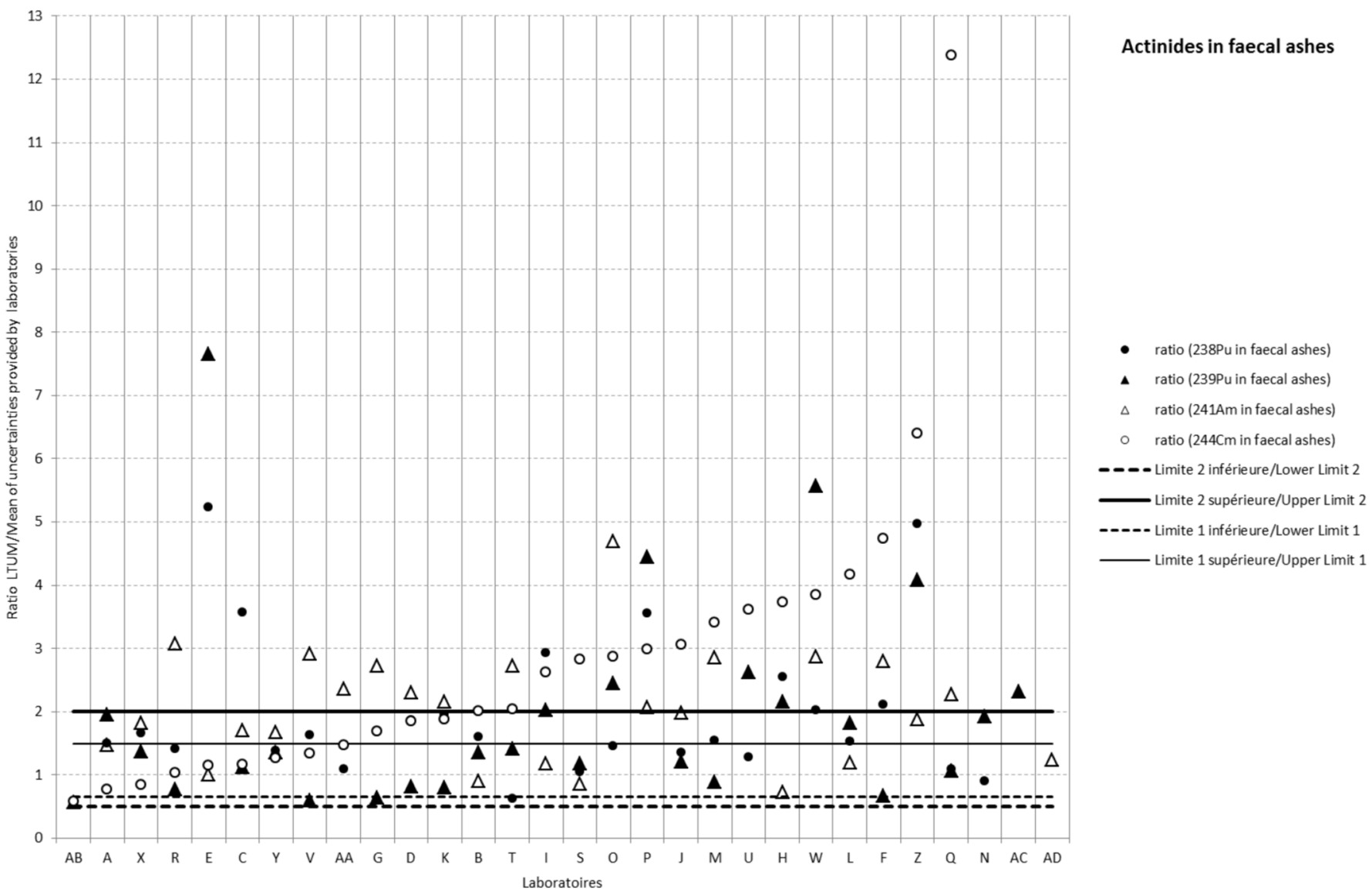

Fig. 10. Rapports entre l'incertitude LTUM et l'incertitude moyenne fournie par les laboratoires (actinides dans les cendres de selles).

Fig. 10. Ratio between LTUM uncertainties and average uncertainties provided by laboratories (actinides in fecal ashes).

étape de comptage et d'analyse de spectre avec ou sans déconvolution qui est très dépendant de l'opérateur. Les résultats obtenus sont le reflet de l'état de l'art car ils ont été obtenus en utilisant les résultats d'un nombre significatif de laboratoires exerçant dans le domaine de la radiotoxicologie ou des mesures de radioactivité bas niveau dans des échantillons de l'environnement. Par contre, il n'y a pas eu de calcul précis en fonction des méthodes pour avoir un classement par groupes de pairs. C'est une étude qui pourra être faite dans un second temps. De plus, les limites acceptables proposées ne concernent que les éléments ou groupe d'éléments pour une matrice donnée. Il est nécessaire de poursuivre les études sur les émetteurs $\alpha$ dans les urines, ${ }^{210}$ Po et l'uranium compris en séparant les deux principes de mesure, de l'activité ou de la masse. De la même manière, l'estimation des incertitudes par la méthode LTUM devra être réalisée pour les autres émetteurs $\beta$ comme le ${ }^{14} \mathrm{C}$ dans les urines, dont le principe de mesure est proche de celui du ${ }^{3} \mathrm{H}, \mathrm{le}^{35} \mathrm{~S}$ et le ${ }^{90} \mathrm{Sr}$. Une étude plus précise pourrait être menée pour les émetteurs $\gamma / X$ dans les urines en faisant une distinction entre les émetteurs $\gamma$ et les émetteurs $X$ comme les iodes.

En conclusion, cette étude a permis de valider le calcul d'incertitude par une méthode simple et pratique utilisant uniquement les résultats des programmes d'intercomparaisons et de proposer des limites acceptables de valeurs d'incertitudes comme dans la plupart des domaines de la biologie médicale grâce au retour d'expérience d'un organisme réalisant des essais d'intercomparaisons dans le domaine de la radiotoxicologie.

\section{Références}

Charki A, et al. 2012. Incertitudes de mesure des radionucléides émetteurs alpha par spectrométrie. In : Incertitude de mesures. Application concrètes pour les essais. Tome 2, pp. 1-12.

CLSI. 2010. Characterization and qualification of commutable reference materials for laboratory medicine. CLSI document EP30-A. Wayne, PA: Clinical and Laboratory Standards Institute.

Cofrac. 2011. SH GTA 14 guide technique d'accréditation pour l'évaluation des incertitudes de mesure en biologie médicale. Révision : octobre 2011. Disponible sur www.cofrac.fr.

EUROLAB. 2007. Measurement uncertainty revisited: alternative approaches to uncertainty evaluation. Technical Report No. 1/ 2007. European Federation of National Associations of Measurement, Testing and Analytical Laboratories, EUROLAB.

GEHT. 2014. Normes d'acceptabilité en hémostase. Propositions du groupe de travail du GEHT. Disponible sur http://site.geht.org/ UserFiles/file/pratiques-professionnelles/Normes_acceptables_he mostase_GE HT2014.pdf.

Ishikawa K. 1980. QC circle Koryo: general principles of the QC circle. Tokyo: Qc Circle Headquarters, Union of Japanese Scientists and Engineers, original Japanese edition 1970. 
ISO. 2005. NF EN ISO/CEI 17025 : exigences générales concernant la compétence des laboratoires d'étalonnage et d'essais.

ISO. 2008. Guide to the expression of uncertainty in measurement. Geneva: International Organization for Standardization.

ISO. 2010a. NF ISO 11929 : détermination des limites caractéristiques (seuil de décision, limite de détection et extrémités de l'intervalle de confiance) pour mesurages de rayonnements ionisants - Principes fondamentaux et applications.

ISO. 2010b. ISO 28218 : radiation protection - Performance criteria for radiobioassay.

ISO. 2012. NF EN ISO 15189 : laboratoires de biologie médicale. Exigences concernant la qualité et la compétence.

ISO. 2015. NF ISO 13528 : méthodes statistiques utilisées dans les essais d'aptitude par comparaisons interlaboratoires.

Matar G, et al. 2015. Uncertainty in measurement for 43 biochemistry, immunoassay, and hemostasis routine analytes evaluated by a method using only external quality assessment data. Clin. Chem. Lab. Med. 53: 1725-1736.

Meijer P, et al. 2002. Long-term analytical performance of hemostasis field methods as assessed by evaluation of the results of an external quality assessment program for antithrombin. Clin. Chem. 48: 1011-1015.

Ministère du Travail, des Relations sociales, de la Famille, de la Solidarité et de la Ville. 2009. Arrêté du 15 décembre 2009 relatif aux contrôles du respect des valeurs limites biologiques fixées à l'article R. 4412-152 du Code du travail pour les travailleurs exposés au plomb et à ses composés et aux conditions d'accréditation des laboratoires chargés des analyses.

Ricos C, et al. 1999. Current database on biologic variation: pros, cons and progress. Scand. J. Clin. Lab. Invest. 59: 491-500. Available from https://www.westgard.com/biodatabase1.htm, mise à jour 2014.

Ritt J, et al. 2001. Protocole de mesure directe de l'uranium dans les urines par ICP MS. Radioprotection 36: 167-181.

Tymen $\mathrm{H}$, et al. 1998. Méthode de dosage du technétium 99 dans les urines par scintillation liquide. Radioprotection 33: 147158.

Vassault A, et al. 1999. Analyses de biologie médicale : spécifications et normes d'acceptabilité à l'usage de la validation de techniques. Ann. Biol. Clin. 57: 685-695.

Citation de l'article : Guichet C, Hurtgen C. 2017. Détermination de limites acceptables pour les incertitudes en radiotoxicologie par une méthode utilisant les résultats d'évaluation externe de la qualité. Radioprotection 52(2): 125-139 